\title{
Aspectos ecológicos de Anopheles cruzii e Culex ribeirensis (Diptera, Culicidae) da Mata Atlântica de Morretes, Paraná, Brasil
}

\author{
Luiz Gonzaga dos Santos-Neto ${ }^{1} \&$ Ana Leuch Lozovei ${ }^{2}$
}

\author{
${ }^{1}$ Departamento de Zoologia, Universidade Federal do Paraná, Centro Politécnico. Caixa Postal 19020, 81531-980 Curitiba-PR, Brasil. \\ luizgonzaga3@hotmail.com \\ ${ }^{2}$ Departamento de Patologia Básica, Universidade Federal do Paraná, 81531-990 Curitiba-PR, Brasil. alozovei@ufpr.br
}

\begin{abstract}
Ecological aspects of Anopheles cruzii and Culex ribeirensis (Diptera, Culicidae) in Atlantic Forest of Morretes, Paraná, Brazil. A study of adult mosquitoes was performed from April 1995 to March 1996 in the Atlantic Forest near to Morretes city, Paraná, Brazil. The research was carried out by using two CDC-M light traps installed in different vertical stratification levels. Mosquitoes were collected monthly throughout the year from 6 p.m. to 6 a.m., with total work hours of 144. A number of 1,408 specimens of mosquitoes was obtained (409 at canopy level and 999 near the ground), belonging to 10 genera and 31 species. Anopheles cruzii and Culex ribeirensis were predominant and are the main subjects of the present study. Difference was not observed between the traps for Anopheles cruzii. However, the higher number of Culex ribeirensis was collected in CDC-M/ground. According to time intervals, Anopheles cruzii showed peaks in the early hours of the night, and decreased progressively until dawn, without a secondary peak. As for the monthly distribution, Anopheles cruzii was more frequent in April and May 1995 and March 1996. Correlations between specimens and temperature or rainfall were not significant. The flight activity of Culex ribeirensis was more intense from 10 p.m. until 4 a.m., but significant peaks were not observed. The monthly distribution of Culex ribeirensis showed peaks in December and January. Significant correlation of this culicine was found for temperature and rainfall. First records for Paraná State: Ochlerotatus rhyacophilus, Culex misionensis, Culex pedroi, Culex ribeirensis and Culex zeteki.
\end{abstract}

KEYWORDS. Ecology; light trap; surveillance.

RESUMO. Aspectos ecológicos de Anopheles cruzii e Culex ribeirensis (Diptera, Culicidae) da Mata Atlântica de Morretes, Paraná, Brasil. Um estudo foi realizado sobre mosquitos adultos de abril 1995 a março 1996 na Mata Atlântica do município de Morretes, Paraná, Brasil. A investigação foi procedida com auxílio de duas armadilhas luminosas CDC-M instaladas em estratos verticais diferentes. Os mosquitos foram capturados mensalmente durante um ano, com início às 18 horas e término às 6 horas do dia seguinte, totalizando 144 horas de trabalho de campo. Obteve-se 1.408 exemplares de mosquitos (409 na copa e 999 próximo ao solo), pertencentes a 10 gêneros e 31 espécies. Anopheles cruzii e Culex ribeirensis foram predominantes e são objetos do presente estudo. Não foi observada diferença entre as armadilhas para Anopheles cruzii. Mas Culex ribeirensis foi coletado em maior número pela CDC-M/solo. Anopheles cruzii, quanto à freqüência horária, apresentou picos nas primeiras horas da noite, depois a sua atividade decresceu progressivamente até o crepúsculo matutino, sem apresentar um pico secundário. Em referência a distribuição mensal, Anopheles cruzii foi mais frequiente nos meses de abril e maio de 1995 e março de 1996. Não houve correlação do número de exemplares com a temperatura ou precipitações pluviométricas. Culex ribeirensis apresentou maior atividade de vôo de $22 \mathrm{~h}$ às $4 \mathrm{~h}$, mas não houve picos significativos. Nas coletas obtidas por mês, Culex ribeirensis teve picos em dezembro e janeiro. Houve correlação do número de espécimes deste culicíneo com a temperatura e precipitações pluviométricas. Constituem os primeiros registros para o Estado do Paraná: Ochlerotatus rhyacophilus, Culex misionensis, Culex pedroi, Culex ribeirensis e Culex zeteki.

PALAVRAS-CHAVE. Armadilha luminosa; ecologia; levantamento.

Os aspectos ecológicos sobre mosquitos, nos seus mais diferentes pontos de vista, são de grande importância para a saúde pública, serviços sanitários e epidemiológicos, pois são dípteros com grande potencialidade para transmitir uma série de agentes patogênicos, como os protozoários causadores da malária humana, nematódeos como Wuchereria e vírus que são responsáveis por doenças como febre amarela, dengue e encefalomielites.

Nas regiões sudeste e sul do Brasil verifica-se grande alteração na paisagem decorrente dos processos de urbanização e exploração inadequada do ambiente natural, com destruição de grandes áreas de florestas. Consequentemente, insetos hematófagos com plasticidade adaptativa para o ambiente de periferia das cidades e a domiciliação podem constituir-se em sérias pragas, além de veiculadores de agentes etiológicos de doenças para as populações humanas e animais (Forattini 1980; Forattini et al. 1989, 1991; Walsh et al. 1993; Walter 2003). Nas supracitadas regiões, as áreas que ainda conservam caracteres de "mata primitiva" estão localizadas principalmente ao longo da Serra do Mar e planície litorânea, tradicionalmente denominada de Mata Atlântica.

Quanto aos estudos da fauna culicideana de Mata Atlântica, alguns dos trabalhos realizados são de Guimarães \& Arlé (1984), Guimarães et al. (1985) e Guimarães \& Victório 
(1986) no Parque Nacional da Serra dos Órgãos, Estado do Rio de Janeiro, e Forattini et al. (1986a, 1986b) no Vale do Ribeira, Estado de São Paulo. Outros trabalhos de Forattini et al. (1968, 1981, 1987, 1989, 1991, 1993a, 1993b) foram realizados em diversas áreas do sul do Estado de São Paulo, e ainda os de Lourenço-de-Oliveira (1984), Lourenço-de-Oliveira \& Silva (1985) e Lourenço-de-Oliveira et al. (1985) nas Granjas Calábria do Rio de Janeiro. Essas referências tratam de mosquitos de áreas modificadas, porém adjacentes e influenciadas, em maior ou menor grau, pela Mata Atlântica.

No Paraná, as espécies silvestres de mosquitos da Mata Atlântica da Serra do Mar e de sua encosta atlântica, incluindo planície litorânea, recentemente foram pesquisadas por Lozovei (2001), Sant'Ana \& Lozovei (2001) e Silva et al. (2004).

O conhecimento mais detalhado de culicídeos da Mata Atlântica trará subsídios para o avanço nos estudos taxonômicos, ecológicos e de distribuição geográfica destes dípteros, além de futuros estudos epidemiológicos de possíveis agentes etiológicos de doenças. O presente estudo teve por objetivo o levantamento da fauna de culicídeos em coletas mensais durante um ano numa área restrita de Mata Atlântica, a fim de verificar em dois estratos verticais a atividade noturna de espécies mais freqüentes. Ao mesmo tempo conferir a distribuição mensal e horária das espécies predominantes em decorrência de variáveis meteorológicas de temperatura e pluviosidade.

\section{MATERIALE MÉTODOS}

O trabalho foi realizado na Estação II do Instituto Agronômico do Paraná (IAPAR), área localizada cerca de $6 \mathrm{~km}$ a noroeste de Morretes (latitude $25^{\circ} 30 \mathrm{~S}$; longitude $48^{\circ} 49 \mathrm{~W}$, $10 \mathrm{~m}$ de altitude) e $2 \mathrm{~km}$ ao sul do distrito de Porto de Cima na planície litorânea do Estado do Paraná. O acesso é feito pela Rodovia Estadual 411 que faz a ligação entre a cidade e o distrito. Essa rodovia é continuação da Rodovia Estadual 410, mais conhecida como Estrada da Graciosa.

A Fazenda Experimental Estação II do IAPAR apresenta, entre outras alterações antrópicas, áreas experimentais de arroz irrigado e pastagens para gado bufalino. Essas áreas estão dominadas por gramíneas e o terreno é freqüentemente encharcado por chuvas e aparente baixo escoamento. Há também brejos temporários e áreas sempre alagadas, formando diversos ambientes propícios a proliferação de mosquitos.

$\mathrm{O}$ local de pesquisa propriamente dito fica localizado no sopé da Serra do Marumbi, na Serra do Mar, que é parte do sistema orográfico do Brasil Oriental na Região Sul, com ponto culminante o Pico Marumbi. Trata-se de Floresta Pluvial Tropical Atlântica ou Floresta Ombrófila Densa, com árvores que alcançam entre 20 e $25 \mathrm{~m}$ de altura. Entre os componentes herbáceos, as famílias de maior freqüência são Bromeliaceae e Araceae (Negrelle 2006).

No que se refere às capturas de mosquitos, foram instaladas em estratos verticais diferentes duas armadilhas CDC-modificadas (CDC-M), conforme Natal et al. (1991), afixadas em galhos de árvores. A armadilha do estrato de copa de árvore ficou a $7 \mathrm{~m}$ de altura (CDC-M/copa). A outra foi instalada a 1,5 m de altura (CDC-M/solo) num arbusto aparente. As árvores que sustentavam as CDC-M distavam entre si em linha horizontal $5 \mathrm{~m}$. O trabalho de coleta ocorreu no interior da floresta, cerca de $200 \mathrm{~m}$ da orla (Marcondes et al. 2001) e em terreno inclinado.

As atividades de coleta estenderam-se de abril de 1995 até março de 1996, com 12 horas noturnas de trabalho, sendo o início às 18 horas e término às 6 horas do dia seguinte, em noite de lua nova, totalizando 144 horas. De duas em duas horas os mosquitos foram recolhidos, trocando os recipientes coletores e, nos mesmos intervalos de tempo, dados de temperatura coletados em termômetro (Incotherm ${ }^{\circledR}$ ). As informações sobre precipitação pluviométrica mensal foram fornecidas pelo Sistema Meteorológico do Paraná (SIMEPAR).

As capturas nas noites de lua nova ocorreram em 27-28/ abril, 30-31/maio, 25-26/junho, 26-27/julho, 24-25/agosto, 2526/setembro, 23-24/outubro, 22-23/novembro, 21-22/ dezembro de 1995 e 19-20/janeiro, 19-20/ fevereiro e 18-19/ março de 1996. A opção pelas noites de lua nova foi estabelecida em vista de maior eficiência das armadilhas luminosas (Provost 1959).

Análise Estatística. Foram feitas análises estatísticas do número de indivíduos das espécies predominantes, conforme os intervalos horários e coletas mensais nas CDC-M/copa e $\mathrm{CDC}-\mathrm{M} /$ solo. Os testes não-paramétricos (Kruskal-Wallis) foram utilizados ao nível de significância de $p$-valor $<0,05$. Para a análise das influências de temperaturas noturnas de coletas e das precipitações pluviométricas mensais sobre a população de adultos, fizeram-se cálculos do coeficiente de correlação de Spearman $\left(r_{s}\right)$. O método de Spearman também foi usado na comparação de contagem das espécies mais freqüentes capturadas em ambas as armadilhas. As análises da presente pesquisa foram efetuadas utilizando o pacote estatístico "R" (http://cran-r.c3sl.ufpr.br/).

\section{RESULTADOS}

Durante 12 meses consecutivos de trabalho de campo foram capturados 1.408 espécimes de mosquitos de 10 gêneros, 31 espécies e mais cinco grupos de espécies. Os machos ficaram representados por apenas 56 espécimes, sendo a maioria coletado pela CDC-M/copa.

Anopheles (Kerteszia) cruzii Dyar \& Knab 1908 e Culex (Melanoconion) ribeirensis Forattini \& Sallum 1985 foram as espécies mais numerosas. A primeira ficou representada por 617 espécimes $(43,82 \%$ do total) e sua coleta nas CDC-M/ copa e CDC-M/solo foi equivalente. Culex ribeirensis teve maior atividade de vôo próximo ao solo, com 157 espécimes capturados na CDC-M/solo. Apenas 10 espécimes foram obtidos na CDC-M/copa. Culex ribeirensis representou 11,86\% no total das espécies coletadas (Tabela I).

Poucos exemplares foram obtidos de outras espécies como Culex (Melanoconion) misionensis Duret 1953 (4,12\% do total), Ochlerotatus rhyacophilus (Costa Lima 1933) (1,99\%), 
Tabela I. Número total de mosquitos coletados nas CDC-M/copa e CDC-M/solo na Mata Atlântica de Morretes, Paraná, Brasil, em abril de 1995 a março de 1996.

\begin{tabular}{|c|c|c|c|c|c|c|}
\hline \multirow{2}{*}{ Espécies } & \multicolumn{2}{|c|}{ CDC-M/copa } & \multicolumn{2}{|c|}{ CDC-M/solo } & \multirow{2}{*}{ TOTAL } & \multirow{2}{*}{$\%$} \\
\hline & $0^{x}$ & ㅇ & $0^{x}$ & 우 & & \\
\hline Aedeomyia (Aedeomyia) squamipennis & - & 3 & - & - & 3 & 0,21 \\
\hline Anopheles (Anopheles) eiseni & - & 1 & - & 2 & 3 & 0,21 \\
\hline Anopheles (Anopheles) fluminensis & - & 4 & - & 2 & 6 & 0,43 \\
\hline Anopheles (Anopheles) intermedius & - & 1 & - & - & 1 & 0,07 \\
\hline Anopheles (Anopheles) mediopunctatus & - & 1 & - & 4 & 5 & 0,36 \\
\hline Anopheles (Kerteszia) cruzii & 14 & 275 & 1 & 327 & 617 & 43,82 \\
\hline Anopheles (Kerteszia) homunculus & - & - & 2 & 1 & 3 & 0,21 \\
\hline Anopheles (Nyssorhynchus) evansae & 1 & - & - & - & 1 & 0,07 \\
\hline Anopheles (Nyssorhynchus) lutzii & - & 1 & - & 1 & 2 & 0,14 \\
\hline Anopheles (Nyssorhynchus) parvus & - & 1 & - & - & 1 & 0,07 \\
\hline Coquillettidia (Rhynchotaenia) chrysonotum & 1 & 2 & - & 4 & 7 & 0,50 \\
\hline Coquillettidia (Rhynchotaenia) nigricans & - & 1 & - & - & 1 & 0,07 \\
\hline Coquillettidia (Rhynchotaenia) venezuelensis & 2 & 7 & - & 18 & 27 & 1,92 \\
\hline Culex (Culex) dolosus & 2 & 4 & - & 4 & 10 & 0,71 \\
\hline Culex (Culex) grupo Coronator & - & 1 & - & 18 & 19 & 1,35 \\
\hline Culex (Culex) nigripalpus & - & 2 & - & 1 & 3 & 0,21 \\
\hline Culex (Culex) spp. & 2 & 18 & 1 & 199 & 220 & 15,63 \\
\hline Culex (Melanoconion) misionensis & 4 & 2 & - & 52 & 58 & 4,12 \\
\hline Culex (Melanoconion) pedroi & - & 1 & - & 1 & 2 & 0,14 \\
\hline Culex (Melanoconion) ribeirensis & - & 10 & - & 157 & 167 & 11,86 \\
\hline Culex (Melanoconion) spp. & 4 & 12 & 2 & 113 & 131 & 9,30 \\
\hline Culex (Melanoconion) zeteki & - & - & 1 & 16 & 17 & 1,21 \\
\hline Culex (Microculex) spp. & 8 & 4 & 8 & 5 & 25 & 1,78 \\
\hline Culex ocellatus (subgênero incerto) & 1 & - & - & - & 1 & 0,07 \\
\hline Ochlerotatus (Ochlerotatus) rhyacophilus & - & 9 & - & 19 & 28 & 1,99 \\
\hline Ochlerotatus (Ochlerotatus) scapularis & - & - & - & 1 & 1 & 0,07 \\
\hline Ochlerotatus (Ochlerotatus) serratus & 1 & 3 & - & 15 & 19 & 1,35 \\
\hline Ochlerotatus (Protomacleaya) terrens & - & 2 & - & 6 & 8 & 0,57 \\
\hline Psorophora (Grabhamia) cingulata & - & - & - & 3 & 3 & 0,21 \\
\hline Psorophora (Janthinosoma) pseudomelanota & - & - & 1 & - & 1 & 0,07 \\
\hline Runchomyia (Runchomyia) reversa & - & 2 & - & 2 & 4 & 0,28 \\
\hline Runchomyia (Runchomyia) spp. & - & - & - & 6 & 6 & 0,43 \\
\hline Shannoniana fluviatilis & - & - & - & 1 & 1 & 0,07 \\
\hline Trichoprosopon pallidiventer & - & - & - & 1 & 1 & 0,07 \\
\hline Uranotaenia (Uranotaenia) calosomata & - & 2 & - & 3 & 5 & 0,36 \\
\hline Uranotaenia (Uranotaenia) nataliae & - & - & - & 1 & 1 & 0,07 \\
\hline Subtotal & 40 & 369 & 16 & 983 & & \\
\hline TOTAL & \multicolumn{2}{|c|}{409} & \multicolumn{2}{|c|}{409} & 1408 & 100,00 \\
\hline
\end{tabular}

Legenda: - ausência; $\$$ Culex dolosus e/ou Culex eduardoi; $\rightarrow$ incluem Culex declarator e Culex mollis.

Coquillettidia venezuelensis (Theobald 1912) (1,92\%), Ochlerotatus serratus (Theobald 1901) (1,35\%) e Culex (Melanoconion) zeteki Dyar 1918 (1,21\%). Estas cinco espécies foram coletadas em maior número pela $\mathrm{CDC}-\mathrm{M} /$ solo, especialmente Culex zeteki, que ficou ausente na CDC-M/ copa. Cinco grupos necessitam de mais estudos para uma diferenciação específica, sendo quatro de Culex. O mais numeroso foi Culex (Culex) $(15,63 \%$ do total), seguido por Culex (Melanoconion) (9,30\%), Culex (Microculex) $(1,78 \%)$ e grupo Coronator de Culex (Culex) (1,35\%). O último destes grupos foi Runchomyia (Runchomyia) (0,43\%). Todos, exceto Culex (Microculex), foram coletados em maior número pela CDC-M/solo, e Runchomyia (Runchomyia) foi coletada apenas nesta armadilha. Para Culex (Microculex), a coleta na CDC$\mathrm{M} /$ copa e CDC-M/solo foi equivalente. Todas as outras espécies ficaram representadas por menos de 1,00\% cada. Numa comparação geral, a CDC-M/solo superou a CDC-M/ copa tanto em número de exemplar capturados como de espécies de mosquitos (Tabela I). Anopheles cruzii e Culex ribeirensis por terem sido as espécies predominantes foram estudadas mais detalhadamente.

Anopheles (Kerteszia) cruzii. Os espécimes de A. cruzii capturados pelas CDC-M/copa e CDC-M/solo por intervalos horários e nas coletas mensais são apresentados resumidamente na Tabela II e os dados de temperaturas e 
Tabela II. Número total de Anopheles cruzii coletados nas CDC-M/copa e CDC-M/solo em distribuição mensal e por intervalos horários na Mata Atlântica de Morretes, Paraná, Brasil, em abril de 1995 a março de 1996.

\begin{tabular}{|c|c|c|c|c|c|c|c|c|c|c|c|c|c|}
\hline & \multicolumn{2}{|c|}{ 18-20h } & \multicolumn{2}{|c|}{$20-22 \mathrm{~h}$} & \multicolumn{2}{|c|}{ 22-0h } & \multicolumn{2}{|c|}{$0-2 \mathrm{~h}$} & \multicolumn{2}{|c|}{$2-4 h$} & \multicolumn{2}{|c|}{ 4-6h } & \multirow{2}{*}{ TOTAL } \\
\hline & copa & solo & copa & solo & copa & solo & copa & solo & copa & solo & copa & solo & \\
\hline Abril & 13 & 53 & 1 & 18 & 1 & 14 & - & 2 & 1 & 1 & 1 & 3 & 108 \\
\hline Maio & 141 & 29 & 16 & 4 & 13 & 2 & 1 & - & 1 & - & - & - & 207 \\
\hline Junho & - & - & 1 & - & - & - & - & - & - & - & - & - & 1 \\
\hline Julho & 6 & 13 & 4 & 2 & 1 & 2 & 2 & - & - & - & - & - & 30 \\
\hline Agosto & 2 & 4 & 3 & 2 & - & - & 1 & - & - & - & 1 & - & 13 \\
\hline Setembro & - & - & - & - & - & - & - & - & - & - & - & - & 0 \\
\hline Outubro & - & - & 1 & - & 1 & - & - & - & - & - & - & - & 2 \\
\hline Novembro & - & - & 1 & - & - & - & - & - & - & - & - & - & 1 \\
\hline Dezembro & - & - & 7 & 1 & - & - & - & 1 & - & 1 & - & - & 10 \\
\hline Janeiro & - & 1 & 1 & 6 & - & - & 1 & - & 1 & 2 & - & - & 12 \\
\hline Fevereiro & - & - & 3 & 1 & - & - & - & - & - & - & - & - & 4 \\
\hline Março & 54 & 158 & 5 & 3 & 1 & 1 & 1 & 1 & 2 & 2 & - & 1 & 229 \\
\hline TOTAL & 216 & 258 & 43 & 37 & 17 & 19 & 6 & 4 & 5 & 6 & 2 & 4 & 617 \\
\hline
\end{tabular}

precipitações pluviométricas em valores acumulados no mês na Tabela IV.

Foram feitas correlações de números de espécimes de $A$. cruzii coletados pela CDC-M/copa e CDC-M/solo. O coeficiente de Spearman indicou, neste caso, alta correlação positiva $\left(r_{s}=0,69 ; p\right.$-valor $\left.<0,0001\right)$. Isso significa que as armadilhas CDC-M/copa e CDC-M/solo apresentaram desempenhos equivalentes. Por isso, os números de $A$. cruzii foram analisados em conjunto no teste de Kruskal-Wallis, quanto aos intervalos horários e as coletas mensais, e no cálculo do coeficiente de correlação de $\operatorname{Spearman}\left(r_{s}\right)$, quanto às influências das temperaturas noturnas e precipitações pluviométricas mensais.

As correlações de temperaturas e números resultantes de A. cruzii mostraram que não houve influência, ao menos direta, deste fator ambiental sobre os espécimes capturados $\left(r_{s}=0,16\right.$; $p$-valor $=0,16)$. Também não foi constatada correlação com a pluviosidade $\left(r_{s}=-0,11 ; p\right.$-valor $\left.=0,72\right)$.

Nos resultados obtidos por intervalos horários, verificouse maior freqüência de $A$. cruzii entre 18 e $20 \mathrm{~h}$, com número médio de 39,50 indivíduos. Pelo teste de Kruskal-Wallis houve diferença significativa entre os intervalos horários $(p$-valor $=$ $0,009)$. O horário de 20-22h apresentou número médio de 6,67 exemplares, e foi o segundo intervalo mais expressivo. Portanto, Anopheles cruzii apresenta picos de atividade de vôo nas primeiras horas da noite.

Com relação às coletas mensais também houve diferença significativa ( $p$-valor $=0,0001)$. Pode-se observar claramente a maior freqüência de A. cruzii em abril e maio (1995) e março (1996) (Tabela II).

Culex (Melanoconion) ribeirensis. C. ribeirensis foi a segunda espécie em número de exemplares coletados. Quase todos os espécimes foram obtidos pela CDC-M/solo, isto é, mais de $90 \%$ em comparação com a CDC-M/copa (Tabelas I). A freqüência por intervalos horários e as coletas mensais de
C. ribeirensis obtidos nas CDC-M/copa e CDC-M/solo constam na Tabela III. Devido ao pequeno número de exemplares capturados pela CDC-M/copa, não foi possível correlacionar as duas armadilhas. Confrontando os números de exemplares coletados na CDC-M/solo com os dados meteorológicos (Tabela IV) houve correlação com a temperatura $\left(r_{s}=0,47 ; p\right.$-valor $\left.<0,0001\right)$ e também com as precipitações pluviométricas mensais $\left(r_{s}=0,66 ; p\right.$-valor $\left.=0,01\right)$.

Quanto aos números de espécimes coletados por intervalos horários, obteve-se maior freqüência de $C$. ribeirensis nas 22-0h, 0-2h e 2-4h, mas no teste de Kruskal-Wallis não houve diferenças significativas ( $p$-valor $=0,66)$. Diferentemente, em relação às coletas mensais, os números de $C$. ribeirensis apresentaram-se significativos ( $p$-valor $=0,001)$. Dezembro foi o mês mais produtivo em comparação a todos os outros meses, com média de 16,5 exemplares na CDC-M/solo. Janeiro foi o segundo mês com média de 5,33 indivíduos. Em outros meses este culicíneo foi coletado com números inexpressivos.

\section{DISCUSSÃO}

Anopheles (Kerteszia) cruzii. Dentre as espécies de Kerteszia, Anopheles cruzii é provavelmente a mais investigada. Vários trabalhos foram desenvolvidos tratando de seus aspectos ecológicos e de comportamentos. É uma espécie bem conhecida como vetor primário do Plasmodium vivax da malária humana no litoral das regiões sudeste e sul do Brasil (Deane et al. 1984; Forattini et al. 1986a, 1986b, 1990, 1993a, 1996; Tubaki et al. 1993; Branquinho et al. 1997). Também é uma espécie suspeita na veiculação de Plasmodium simium e Plasmodium brasilianum, causadores da malária simiana (Deane et al. 1984; Deane 1992; Curado et al. 1997), mas ainda não confirmada, de acordo com revisão recente (Nunn \& Heymann 2005).

Anopheles cruzii normalmente é a espécie predominante entre outras do gênero no interior da Mata Atlântica. Este fato 
Tabela III. Número total de Culex ribeirensis coletados nas CDC-M/copa e CDC-M/solo em distribuição mensal e por intervalos horários na Mata Atlântica de Morretes, Paraná, Brasil, em abril de 1995 a março de 1996.

\begin{tabular}{|c|c|c|c|c|c|c|c|c|c|c|c|c|c|}
\hline & \multicolumn{2}{|c|}{$18-20 \mathrm{~h}$} & \multicolumn{2}{|c|}{$20-22 \mathrm{~h}$} & \multicolumn{2}{|c|}{$22-0 \mathrm{~h}$} & \multicolumn{2}{|c|}{$0-2 \mathrm{~h}$} & \multicolumn{2}{|c|}{$2-4 \mathrm{~h}$} & \multicolumn{2}{|c|}{ 4-6h } & \multirow{2}{*}{ TOTAL } \\
\hline & copa & solo & copa & solo & copa & solo & copa & solo & copa & solo & copa & solo & \\
\hline Abril & - & 1 & - & - & - & 1 & - & - & - & 1 & - & - & 3 \\
\hline Maio & - & - & - & - & - & - & - & - & - & - & - & - & 0 \\
\hline Junho & - & - & - & - & - & - & - & - & - & - & - & - & 0 \\
\hline Julho & - & 3 & - & - & - & - & - & - & - & - & - & - & 3 \\
\hline Agosto & 1 & - & - & 2 & 1 & - & - & 1 & - & 1 & - & - & 6 \\
\hline Setembro & - & - & - & - & - & - & - & - & - & - & - & 1 & 1 \\
\hline Outubro & - & - & 1 & - & - & - & - & 1 & - & - & - & - & 2 \\
\hline Novembro & - & - & - & 1 & - & 1 & - & - & - & 1 & - & - & 3 \\
\hline Dezembro & - & - & - & 7 & - & 32 & 2 & 37 & 1 & 16 & - & 7 & 102 \\
\hline Janeiro & - & - & 1 & 9 & - & 5 & - & 9 & 2 & 8 & - & 1 & 35 \\
\hline Fevereiro & - & - & - & - & 1 & 3 & - & - & - & - & - & - & 4 \\
\hline Março & - & 1 & - & 1 & - & - & - & 1 & - & 5 & - & - & 8 \\
\hline TOTAL & 1 & 5 & 2 & 20 & 2 & 42 & 2 & 49 & 3 & 32 & 0 & 9 & 167 \\
\hline
\end{tabular}

foi constatado nas mais variadas técnicas de captura (isca humana, armadilhas de Shannon e CDC). Na pesquisa de Morretes, com armadilhas CDC-M, Anopheles cruzii apresentou atividade de vôo em diferentes horários, mas com picos incluindo parte do crepúsculo vespertino e primeiras horas da noite. Constatou-se também sua atividade durante todos os meses do ano. Houve uma redução de adultos no inverno, que se prolongou na primavera, aparentemente devido aos dias mais frios. Ainda quanto à atividade sazonal, Anopheles cruzii foi mais abundante no outono e no verão, basicamente nos meses de abril e maio (outono de 1995) e março (verão de 1996). Estes dados estão de acordo, de modo geral, com os trabalhos de outros pesquisadores, exceto no fato de que não foi observado pico no horário do crepúsculo matutino (Forattini et al. 1986a, 1986b, 1993a, 1996; Guimarães \& Arlé 1984; Guimarães et al. 1985; Guimarães \& Victório 1986; Gomes et al. 1987; Tubaki et al. 1993).

Em Morretes, observou-se que nos meses de temperaturas mais altas (acima de $20^{\circ} \mathrm{C}$ ) não ocorreu uma "explosão populacional" repentina de Anopheles cruzii. Isso sugere que a população de adultos aumenta de modo lento e gradual, atingindo picos somente em meados de outono, mesmo com os registros de temperaturas mais baixas. O declínio subseqüente de adultos, nos meses considerados mais frios, ocorreu, provavelmente, com o retardo no desenvolvimento das larvas. Tanto na natureza como no laboratório, o desenvolvimento dos imaturos ocorre lentamente (Aragão 1968; Wilkerson \& Peyton 1991; Chahad-Ehlers et al. 2007). No laboratório, Wilkerson \& Peyton (1991) observaram que o desenvolvimento do ovo ao adulto foi de 35 dias. ChahadEhlers et al. (2007), também em pesquisa realizada em laboratório, obtiveram a evolução de estágio de ovo a emergência de adulto em média de 31,17 dias. O desenvolvimento de imaturos mais acelerado é favorecido com dieta adicional, como a ração de peixes ornamentais (TetraMin®). Por outro lado, na natureza, Aragão (1968) verificou que as larvas de pelo menos três espécies de Kerteszia (Anopheles cruzii, Anopheles homunculus e Anopheles bellator) podem demorar mais do que quatro meses para completar o seu desenvolvimento.

Os adultos de Anopheles cruzii normalmente são mencionados como acrodendrófilos (Forattini et al. 1968; Guimarães et al. 1985). Tem capacidade para voar das copas árboreas ao solo e vice-versa em busca de seus hospedeiros (Deane et al. 1984). No presente estudo, Anopheles cruzii teve atividades durante todo o ano nas copas, exceto setembro. Ao passo que nos resultados obtidos pela $\mathrm{CDC}-\mathrm{M} /$ solo não se verificou essa constância, pois em junho, setembro, outubro e novembro nenhum exemplar foi coletado. Todavia, numa análise geral, as armadilhas da copa e a próxima ao solo apresentaram resultados equivalentes. Confrontando os trabalhos de Forattini (1962) e Deane et al. (1984) com a presente pesquisa pode-se inferir que a população local de Anopheles cruzii apresenta uma distribuição na floresta relativamente homogênea.

Culex (Melanoconion) ribeirensis. Este culicíneo foi descrito por Forattini \& Sallum em 1985, mas tem vários registros antigos como Culex epanastasis e Culex crybda. Acredita-se que esteve envolvido na transmissão do vírus da encefalomielite eqüina do leste (EEE) na região sul do Estado de São Paulo, sob a designação de Culex epanastasis (Calisher et al. 1983). C. ribeirensis permaneceu mais tempo identificado erroneamente como Culex crybda em Forattini et al. 1981, Lourenço-de-Oliveira 1984, Lourenço-de-Oliveira et al. 1985, e Lourenço-de-Oliveira \& Silva 1985. Os registros geográficos deste culicíneo incluem os Estados de Rio de Janeiro, São Paulo e Rio Grande do Sul (Forattini \& Sallum 1985; Sallum \& Forattini 1996; Cardoso et al. 2005). Seu registro para o Estado do Paraná é apresentado pela primeira vez neste trabalho.

Em São Paulo é uma espécie comum nas regiões próximas às planícies litorâneas e na encosta da Serra do Mar (Forattini 
Tabela IV. Temperaturas $\left({ }^{\circ} \mathrm{C}\right)$ obtidas nas noites de coletas e precipitações pluviométricas $(\mathrm{mm})$, Morretes, Paraná, Brasil, abril de 1995 a março de 1996. Precip. M.: precipitações pluviométricas em valores acumulados no mês.

\begin{tabular}{|c|c|c|c|c|c|c|c|c|c|c|c|c|}
\hline Horários & abr. & mai. & jun. & jul. & ago. & set. & out. & nov. & dez. & jan. & fev. & mar. \\
\hline 18-20h & 22,0 & 16,5 & 16,5 & 18,0 & 19,5 & 17,0 & 17,5 & 22,5 & 25,5 & 22,5 & 23,5 & 22,5 \\
\hline $20-22 \mathrm{~h}$ & 21,5 & 15,0 & 15,0 & 17,0 & 19,5 & 17,0 & 16,0 & 21,5 & 24,5 & 21,5 & 23,5 & 22,0 \\
\hline 22-0h & 21,0 & 14,0 & 14,0 & 16,0 & 18,5 & 16,5 & 15,0 & 20,5 & 23,5 & 21,0 & 23,5 & 22,0 \\
\hline $0-2 \mathrm{~h}$ & 20,5 & 13,0 & 13,0 & 15,0 & 17,5 & 16,5 & 14,5 & 19,5 & 23,5 & 20,5 & 23,0 & 22,0 \\
\hline $2-4 h$ & 20,0 & 12,5 & 13,0 & 15,0 & 17,5 & 16,5 & 13,5 & 19,0 & 23,0 & 20,0 & 22,5 & 21,5 \\
\hline 4-6h & 18,5 & 11,5 & 13,0 & 14,5 & 17,0 & 16,5 & 13,5 & 18,5 & 23,0 & 20,0 & 22,5 & 21,5 \\
\hline Média & 20,5 & 13,7 & 14,0 & 15,9 & 18,2 & 16,6 & 15,0 & 20,2 & 23,8 & 20,9 & 23,0 & 21,9 \\
\hline Amplitude & 3,5 & 5,0 & 3,5 & 3,5 & 2,5 & 0,5 & 4,0 & 4,0 & 2,5 & 2,5 & 1,0 & 1,0 \\
\hline Precip. M. & 98,9 & 36,7 & 74,5 & 145,6 & 74,2 & 141,8 & 118,6 & 193,6 & 300,0 & 342,8 & 306,6 & 259,6 \\
\hline
\end{tabular}

\& Sallum 1985). Tem capacidade de domiciliação, facilmente adapta-se ao ambiente alterado pelo homem, como as áreas de projetos de colonização implantadas com o desflorestamento, mas ainda cercadas por uma floresta remanescente (Forattini et al. 1986b, 1987, 1991, 1995).

C. ribeirensis tem atividade de vôo noturna, são comumente capturados em número considerável em armadilhas luminosas do tipo Shannon e CDC, além de isca humana (Forattini et al. 1981, 1991, 1993b; Lourenço-de-Oliveira 1984; Lourenço-deOliveira \& Silva 1985). No trabalho de Morretes, em CDC-M, observou-se maior atividade de vôo noturna durante a madrugada. Nos meses de temperaturas mais elevadas, este culicíneo esteve distribuído com certa regularidade durante toda a noite, fato que coincide com os dados de Forattini et. al. (1991).

Em Morretes apresentou um pico de atividades no mês de dezembro e um segundo, um pouco menor, em janeiro, contrastando em parte com os dados de Forattini et. al. (1991), que mencionaram o mês de março como o de maior ocorrência dessa espécie.

Em um ano de trabalho de capturas, com duas armadilhas instaladas em dois estratos verticais, foram obtidos os primeiros registros para o Estado do Paraná as seguintes espécies: Ochlerotatus rhyacophilus (Costa Lima 1933), Culex misionensis Duret 1953, Culex pedroi Sirivanakarn \& Belkin 1980, Culex ribeirensis Forattini \& Sallum 1985 e Culex zeteki Dyar 1918. Este fato indica claramente a necessidade de mais pesquisas na Mata Atlântica Paranaense. O maior conhecimento das espécies de culicídeos que realmente existem nesta área, coletando-os com diferentes armadilhas e métodos, garantirá subsídios para trabalhos futuros de Bioecologia, Sistemática, Taxonomia e comportamento de mosquitos vetores. Levantamento sistemático das espécies de mosquitos, especialmente em uma área que oferece muitos tipos de criadouros, é necessário e deve estar em constante monitoramento para detectar espécies importantes para a saúde pública.

Agradecimentos. À Coordenadoria de Aperfeiçoamento de Pessoal de Nível Superior (CAPES), pela concessão de bolsa de estudo ao primeiro autor. Ao Instituto Agronômico do Paraná (IAPAR), por intermédio do
Sr. Luís Adão, pela permição das coletas na Estação II de Morretes. Ao Sistema Meteorológico do Paraná (SIMEPAR), pelos dados meteorológicos. À Dra. Maria Anice Mureb Sallum da Faculdade de Saúde Pública da Universidade de São Paulo (USP), pela confirmação das espécies e esclarecimentos na identificação de Culex (Melanoconion). À Dra. Samira Chahad-Ehlers pelas sugestões e críticas construtivas ao manuscrito.

\section{REFERÊNCIAS}

Aragão, M. B. 1968. O ciclo anual dos Anopheles do subgênero Kerteszia, no sul do Brasil. Memórias do Instituto Oswaldo Cruz 66: 85106.

Branquinho, M. S.; M. T. Marrelli; I. Curado; D. Natal; J. M. S. Barata; R. Tubaki; G. C. Carréri-Bruno; R. T. de Menezes \& J. K. Kloetzel. 1997. Infecção do Anopheles (Kerteszia) cruzii por Plasmodium vivax e Plasmodium vivax variante VK247 nos Municípios de São Vicente e Juquitiba, São Paulo. Revista Panamericana de Salud Pública 2: 189-193.

Calisher, C. H.; T. L. M. Coimbra; O. de S. Lopes; D. J. Muth; L. de A. Sacchetta; D. B. Francy; J. S. Lazuick \& C. B. Cropp. 1983 Identification of New Guama and Group C serogroup bunyaviruses and an ungrouped virus from southern Brazil. American Journal of Tropical Medicine and Hygiene 32: 424-431.

Cardoso, J. da C.; E. Corseuil \& J. M. S. Barata. 2005. Culicinae (Diptera, Culicidae) ocorrentes no Estado do Rio Grande do Sul, Brasil. Revista Brasileira de Entomologia 49: 275-287.

Chahad-Ehlers, S.; A. L. Lozovei \& M. D. Marques. 2007. Reproductive and post-embryonic daily rhythm patterns of the malaria vector Anopheles (Kerteszia) cruzii: aspects of the life cycle. Chronobiology International 24: 289-304.

Curado, I.; A. M. R. C. Duarte; A. A. Lal; S. G. Oliveira \& J. K. Kloetzel. 1997. Antibodies anti bloodstream and circumsporozoite antigens (Plasmodium vivax and Plasmodium malariae/P. brasilianum) in areas of very low malaria endemicity in Brazil. Memórias do Instituto Oswaldo Cruz 92: 235-243.

Deane, L. M. 1992. Simian malaria in Brazil. Memórias do Instituto Oswaldo Cruz 87: 1-20.

Deane, L. M.; J. A. Ferreira-Neto \& M. Moura-Lima. 1984. The vertical dispersion of Anopheles (Kerteszia) cruzii in a forest in southern Brazil suggests that human cases of malaria of simian origin might be expected. Memórias do Instituto Oswaldo Cruz 79: 461463.

Forattini, O. P. 1962. Entomologia Médica. Volume 1 - Generalidades, Anophelini, Malária. Editora da Universidade de São Paulo, 662 pp.

Forattini, O. P. 1980. Biogeografia, origem e distribuição da domiciliação de triatomíneos no Brasil. Revista de Saúde Pública 14: $265-299$. 
Forattini, O. P.; A. de C. Gomes; I. Kakitani \& D. Marucci. 1991. Observações sobre domiciliação de mosquitos Culex (Melanoconion) em ambiente com acentuadas modificações antrópicas. Revista de Saúde Pública 25: 257-266.

Forattini, O. P.; A. de C. Gomes; D. Natal; I. Kakitani \& D. Marucci. 1987. Preferências alimentares de mosquitos Culicidae no Vale do Ribeira, São Paulo, Brasil. Revista de Saúde Pública 21: 171187.

Forattini, O. P.; A. de C. Gomes; D. Natal; I. Kakitani \& D. Marucci. 1989. Preferências alimentares e domiciliação de mosquitos Culicidae no Vale do Ribeira, São Paulo, Brasil, com especial referência a Aedes scapularis e a Culex (Melanoconion). Revista de Saúde Pública 23: 9-19.

Forattini, O. P.; A. de C. Gomes; D. Natal \& J. L. F. Santos. 1986a. Observações sobre atividade de mosquitos Culicidae em mata primitiva da encosta no Vale do Ribeira, São Paulo, Brasil. Revista de Saúde Pública 20: 1-20.

Forattini, O. P.; A. de C. Gomes; D. Natal \& J. L. F. Santos. 1986b. Observações sobre atividade de mosquitos Culicidae em matas primitivas da planície e perfis epidemiológicos de vários ambientes no Vale do Ribeira, São Paulo, Brasil. Revista de Saúde Pública 20: $178-203$.

Forattini, O. P.; A. de C. Gomes; J. L. F. Santos; E. A. B. Galati; E. X. Rabello \& D. Natal. 1981. Observações sobre atividade de mosquitos Culicidae em mata residual no Vale do Ribeira, São Paulo, Brasil. Revista de Saúde Pública 15: 557-586.

Forattini, O. P.; A. de C. Gomes; J. L. F. Santos; I. Kakitani \& D. Marucci. 1990. Freqüência ao ambiente humano e dispersão de mosquitos Culicidae em área adjacente a Mata Atlântica primitiva da planície. Revista de Saúde Pública 24: 101-107.

Forattini, O. P.; I. Kakitani; E. Massad \& A. de C. Gomes. 1993a. Studies on mosquitoes (Diptera: Culicidae) and anthropic environment. 1. Parity of blood seeking Anopheles (Kerteszia) in southeastern Brazil. Revista de Saúde Pública 27: 1-8.

Forattini, O. P.; I. Kakitani; E. Massad \& D. Marucci. 1993b. Studies on mosquitoes (Diptera: Culicidae) and anthropic environment. 4. Survey of resting adults and synanthropic behaviour in SouthEastern Brazil. Revista de Saúde Pública 27: 398-411.

Forattini, O. P.; I. Kakitani; E. Massad \& D. Marucci. 1996. Studies on mosquitoes (Diptera: Culicidae) and anthropic environment. 11. Biting activity and blood-seeking parity of Anopheles (Kerteszia) in South-Eastern Brazil. Revista de Saúde Pública 30: 107114.

Forattini, O. P.; O. de S. Lopes \& E. X. Rabello. 1968. Investigações sobre o comportamento de formas adultas de mosquitos silvestres no Estado de São Paulo, Brasil. Revista de Saúde Pública 2: $111-173$

Forattini, O. P. \& M. A. M. Sallum. 1985. A new species of Culex (Melanoconion) from southern Brazil (Diptera: Culicidae). Revista de Saúde Pública 19: 171-182.

Forattini, O. P.; M. A. M. Sallum; I. Kakitani; E. Massad \& D. Marucci. 1995. Studies on mosquitoes (Diptera: Culicidae) and anthropic environment. 8. Survey of adult behaviour of Spissipes Section species of Culex (Melanoconion) in South-Eastern Brazil. Revista de Saúde Pública 29: 100-107.

Gomes, A. de C.; O. P. Forattini \& D. Natal. 1987. Composição e atividade de mosquitos Culicidae. Emprego de armadilha CDC no Vale do Ribeira, Estado de São Paulo, Brasil. Revista de Saúde Pública 21: 363-370.

Guimarães, A. E. \& M. Arlé. 1984. Mosquitos no Parque Nacional da Serra dos Órgãos, Estado do Rio de Janeiro, Brasil. 1. Distribuição estacional. Memórias do Instituto Oswaldo Cruz 79: 309323.

Guimarães, A. E.; M. Arlé \& R. N. M. Machado. 1985. Mosquitos no Parque Nacional da Serra dos Órgãos, estado do Rio de Janeiro,
Brasil. 2. Distribuição vertical. Memórias do Instituto Oswaldo Cruz 80: 171-185.

Guimarães, A. E. \& V. M. N. Victório. 1986. Mosquitos no Parque Nacional da Serra dos Órgãos, Estado do Rio de Janeiro, Brasil. 3. Preferência horária para hematofagia. Memórias do Instituto Oswaldo Cruz 81: 93-103.

Lourenço-de-Oliveira, R. 1984. Alguns aspectos da ecologia dos mosquitos (Diptera: Culicidae) de uma área de planície (Granjas Calábria), em Jacarepaguá, Rio de Janeiro. 1. Freqüência comparativa das espécies em diferentes ambientes e métodos de coleta. Memórias do Instituto Oswaldo Cruz 79: 479-490.

Lourenço-de-Oliveira, R. \& T. F. da Silva. 1985. Alguns aspectos da ecologia dos mosquitos (Diptera: Culicidae) de uma área de planície (Granjas Calábria), em Jacarepaguá, Rio de Janeiro. 3. Preferência horária das fêmeas para o hematofagismo. Memórias do Instituto Oswaldo Cruz 80: 195-201.

Lourenço-de-Oliveira, R.; T. F. da Silva \& R. Heyden. 1985. Alguns aspectos da ecologia dos mosquitos (Diptera: Culicidae) de uma área de planície (Granjas Calábria) em Jacarepaguá, Rio de Janeiro. 2. Freqüência mensal e no ciclo lunar. Memórias do Instituto Oswaldo Cruz 80: 123-133.

Lozovei, A. L. 2001. Microhabitats de mosquitos (Diptera, Culicidae) em internódios de taquara na Mata Atlântica, Paraná, Brasil. Iheringia, Série Zoologia 90: 3-13.

Marcondes, C. B.; L. G. dos Santos-Neto \& A. L. Lozovei. 2001. Ecology of Phlebotomine sandflies (Diptera, Psychodidae) in Brazilian Atlantic Forest. Revista da Sociedade Brasileira de Medicina Tropical 34: 255-260.

Natal, D.; D. Marucci; I. M. dos Reis \& E. A. B. Galati. 1991. Modificação da armadilha CDC com testes para coletas de flebotomíneos (Diptera). Revista Brasileira de Entomologia 35: 697-700.

Negrelle, R. R. B. 2006. Composição florística e estrutura vertical de um trecho de Floresta Ombrófila Densa de Planície Quaternária. Hoehnea 33: 261-289.

Nunn, C. L. \& E. W. Heymann. 2005. Malaria infection and host behavior: a comparative study of Neotropical primates. Behavioral Ecology and Sociobiology 59: 30-37.

Provost, M. W. 1959. The influence of moonlight on light-trap catches of mosquitoes. Annals of the Entomological Society of America 52: 261-271.

Sallum, M. A. M. \& O. P. Forattini. 1996. Revision of the Spissipes Section of Culex (Melanoconion) (Diptera: Culicidae). Journal of the American Mosquito Control Association 12: 517-600.

Sant'Ana, A. L. \& A. L. Lozovei. 2001. Influência do ciclo lunar na atividade de vôo de Coquillettidia (Rhynchotaenia) venezuelensis (Theobald) (Diptera, Culicidae) na Mata Atlântica, Serra do Marumbi, Morretes, Paraná, Brasil. Revista Brasileira de Zoologia 18: $365-374$

Silva, A. M. da; V. Nunes \& J. Lopes. 2004. Culicídeos associados a entrenós de bambu e bromélias, com ênfase em Aedes (Stegomyia) albopictus (Diptera, Culicidae) na Mata Atlântica, Paraná, Brasil. Iheringia, Série Zoologia 94: 63-66.

Tubaki, R. M.; G. C. Carréri-Bruno; C. M. Glasser \& R. M. C. Ciaravolo. 1993. Biting activity of Anopheles (Kerteszia) cruzii (Diptera, Culicidae) in domiciliary habitats in the Southern Atlantic Forest, Peruibe, State of Sao Paulo, Brazil. Revista Brasileira de Entomologia 37: 569-575.

Walsh, J. F.; D. H. Molyneux; M. H. Birley. 1993. Deforestation: effects on vector-borne disease. Parasitology 106: S55-S75.

Walter, A. 2003. Activités humaines et trypanosomose américaine: revue de la littérature. Parasite 10: 191-204.

Wilkerson, R. C. \& E. L. Peyton. 1991. The Brazilian malaria vector Anopheles (Kerteszia) cruzii: life stages and biology (Diptera: Culicidae). Mosquito Systematics 23: 110-122. 